\title{
Explorative Analysis of Low-Dose Metronomic Chemotherapy with Cyclophosphamide and Methotrexate in a Cohort of Metastatic Breast Cancer Patients
}

\author{
Slavomir Krajnak Marco Battista Walburgis Brenner Katrin Almstedt Tania Elger \\ Anne-Sophie Heimes Annette Hasenburg Marcus Schmidt
}

Department of Gynecology and Obstetrics, University Medical Center Mainz, Mainz, Germany

\begin{abstract}
Keywords
Adverse events - Breast cancer - Metastasized .

Chemotherapy - Metronomic chemotherapy .

Cyclophosphamide - Methotrexate
\end{abstract}

\section{Summary}

Background: Low-dose metronomic chemotherapy (LDMC) is increasingly used in metastatic breast cancer $(\mathrm{MBC})$. In this retrospective analysis, we examined the therapeutic effects and side effects of LDMC in a cohort of MBC patients. Methods: Patients with MBC were included when LDMC with oral cyclophosphamide (CTX) and methotrexate (MTX) was administered between 2009 and 2015. The primary endpoint was disease control rate $(D C R) \geq 24$ weeks after the start of LDMC. Secondary endpoints were duration of progression-free survival (PFS), rates of discontinuation due to side effects, and DCR with regard to subgroups. Results: Retrospective data of 35 patients were available for this analysis. $31 \%$ patients achieved DCR. The median PFS was 12 weeks. $9 \%$ of patients discontinued LDMC due to adverse events. DCR was $37 \%$ in the first 2 lines and $25 \%$ in further lines of therapy. $22 \%$ of patients with multiple metastases and $35 \%$ with $\leq 2$ different metastatic sites achieved DCR. DCR was achieved in 33\% of hormone receptor(HR)-positive patients and $27 \%$ of HR-negative patients. Conclusion: The DCR of $31 \%$ is in line with the results of previous phase II studies. LDMC was well tolerated. Subgroup analysis was not able to identify a group in which LDMC was more efficient.

(C) 2018 S. Karger GmbH, Freiburg

\section{Introduction}

The concept of conventional chemotherapy is based on the maximum tolerated dose (MTD) given at 2- to 4-week intervals. The aim of this therapy schedule is to affect rapidly dividing cells and to kill as many malignant cells as possible [1]. In addition, non-malignant highly proliferative cells are also affected by these agents. Thus, adverse events like mucositis, myelosuppression, and hair loss are common and represent clinically relevant sequelae [2]. In order to preserve quality of life for patients with advanced cancer, other strategies with fewer side effects have been evaluated [3, 4]. In hormone receptor(HR)-positive metastatic breast cancer $(\mathrm{MBC})$ patients without severe symptoms of disease, endocrine therapy should be applied as an initial therapy. In the case of disease progression or in patients with HR-negative MBC, cytostatic agents with or without targeted therapies are recommended [5].

The daily administration of cytostatic drugs in low doses is called low-dose metronomic chemotherapy (LDMC) $[1,6]$. Many studies showed that LDMC used for palliation can achieve disease control and prolonged overall survival without severe side effects [7]. Over the last decade, clinicians have begun to consider introducing LDMC much earlier into the treatment plan $[8,9]$. In comparison with conventional chemotherapy, the lower doses of LDMC may induce fewer side effects [10-13], which may lead to a lower rate of discontinuation due to side effects such as myelosuppression [10]. In this explorative retrospective analysis, the therapeutic effects and the side effects of LDMC with cyclophosphamide (CTX) and methotrexate (MTX) in patients with MBC were examined. Furthermore, we tried to define a subgroup of patients in which LDMC is more favorable.

\section{KARGER}

() 2018 S. Karger GmbH, Freiburg 
Table 1. Clinical patient characteristics

\begin{tabular}{|c|c|}
\hline Median age at start of treatment (range), years & $64(35-83)$ \\
\hline \multicolumn{2}{|l|}{ Number of current chemotherapy lines, $n$} \\
\hline$\leq 2$ & 19 \\
\hline 1 & 8 \\
\hline 2 & 11 \\
\hline$>2$ (heavily pretreated) & 16 \\
\hline 3 & 10 \\
\hline 4 & 3 \\
\hline 5 & 1 \\
\hline 6 & 1 \\
\hline 7 & 0 \\
\hline 8 & 1 \\
\hline \multicolumn{2}{|l|}{ Number of metastatic sites at start of treatment, $n$} \\
\hline$\leq 2$ & 26 \\
\hline 1 & 13 \\
\hline 2 & 13 \\
\hline$>2$ (multiple metastases) & 9 \\
\hline 3 & 5 \\
\hline 4 & 4 \\
\hline \multicolumn{2}{|l|}{ Metastatic sites, $\mathrm{n}$} \\
\hline Bone & 20 \\
\hline Liver & 17 \\
\hline Lung & 13 \\
\hline Lymph & 9 \\
\hline Pleura & 6 \\
\hline Other (thoracic wall, cutaneous, cerebral, peritoneum) & 6 \\
\hline \multicolumn{2}{|l|}{ Hormone receptor status, $\mathrm{n}$} \\
\hline Positive & 24 \\
\hline Negative & 11 \\
\hline \multicolumn{2}{|l|}{ HER2 status, $n$} \\
\hline Positive & 1 \\
\hline Negative & 34 \\
\hline
\end{tabular}

\section{Patients and Methods}

We screened patients with MBC who received CTX $50 \mathrm{mg}$ daily and MTX $2.5 \mathrm{mg}$ every second day at the Department of Gynecology and Obstetrics of the University Medical Center Mainz between 2009 and 2015. Patient characteristics such as number of chemotherapy lines and different metastatic sites, metastasis location, HR status, and HER2 status were extracted from the patient files. The therapeutic efficacy was determined as disease control rate (DCR) for $\geq 24$ weeks after the start of LDMC medication. DCR included complete remission (CR), partial remission (PR), and stable disease (SD). Progression-free survival (PFS) was defined as the time from the start of therapy to the detection of progressive disease (PD) or death. Duration of response (DoR) was defined as the time from documentation of tumor response to PD. Toxicity was assessed by common terminology criteria for adverse events (CTCAE) version 4.0. For subgroup analysis, we stratified the patients by number of chemotherapy lines (heavily pretreated $\geq 2$ chemotherapy lines), number of different metastatic sites (multiple metastases $\geq 2$ different metastatic sites), and HR and HER2 status. The SPSS statistical software system, version 19.0. was used for statistical analyses (IBM Corp., Armonk, NY, USA)

\section{Results}

Retrospective data of 35 patients were available for this analysis. Patient characteristics are displayed in table 1 . Briefly, the median
Table 2. Treatment response (disease control rate $\geq 24$ weeks)

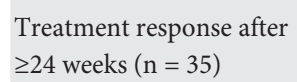

$\geq 24$ weeks $(\mathrm{n}=35)$

Progressive disease

Disease control rate

Stable disease

Partial remission

Complete remission n (\%)

$24(68.6)$

$11(31.4)$

$4(11.4)$

$6(17.1)$

$1(2.9)$
Table 3. Therapy duration, progression-free survival (PFS), and duration of response (DoR)

\begin{tabular}{lllll}
\hline & Mean & Median & Minimum & Maximum \\
\hline Therapy duration, weeks & 26.6 & 12 & 6 & 86 \\
PFS, weeks & 27 & 12 & 6 & 86 \\
DoR, weeks & 36.2 & 22 & 8 & 74 \\
\hline
\end{tabular}

Table 4. Adverse events

\begin{tabular}{ll}
\hline Adverse events $(\mathrm{n}=35)$ & $\mathrm{n}(\%)$ \\
\hline $\begin{array}{l}\text { Cyclophosphamide/methotrexate } \\
\text { (MTX) dropouts }\end{array}$ & $3(8.6)$ \\
Thrombocytopenia & $1(2.9)$ \\
Fatigue & $1(2.9)$ \\
Nausea/vomiting & $1(2.9)$ \\
MTX dropouts & $4(11.4)$ \\
Fatigue & $2(5.7)$ \\
Nausea/vomiting & $2(5.7)$ \\
Visual impairment & $1(2.9)$ \\
Hemorrhagic cystitis & $1(2.9)$ \\
\hline
\end{tabular}

age was 64 years (range $35-83$ years). All patients were pretreated with an endocrine or targeted therapy and/or other cytostatic drugs. The treated cohort received a median of 2 (range 1-8) lines of chemotherapy (including LDMC). A median of 2 (range 1-4) different metastatic sites of $\mathrm{MBC}$ were present; the most frequent location was bone (20 patients), liver (17 patients), and lung (13 patients). 24 patients presented with HR positivity, and 1 patient showed HER2 positivity at diagnosis.

DCR was achieved in 11 (31\%) patients (tables 2, 3). 1 (3\%), 6 (17\%), and 4 (11\%) patients achieved CR, PR, and SD, respectively. The remaining 24 (69\%) patients showed PD. The median PFS was 12 weeks (range 6-86 weeks). Therapy response was documented in 15 (43\%) patients, and the median DoR was 22 weeks (range 8-74 weeks).

During LDMC, discontinuation of therapy due to adverse events occurred (table 4): 3 (9\%) patients receiving CTX/MTX stopped therapy due to intolerable side effects. Thrombocytopenia, gastrointestinal complaints, and fatigue were the most common reasons for early termination of therapy. 4 (11\%) patients stopped only MTX, mainly because of gastrointestinal symptoms and visual impairment. 1 (3\%) patient developed hemorrhagic cystitis but no therapy termination was necessary.

Subgroup analysis did not reveal a specific group of patients who showed benefit from LDMC (table 5, fig. 1). 4/16 (25\%) heav- 

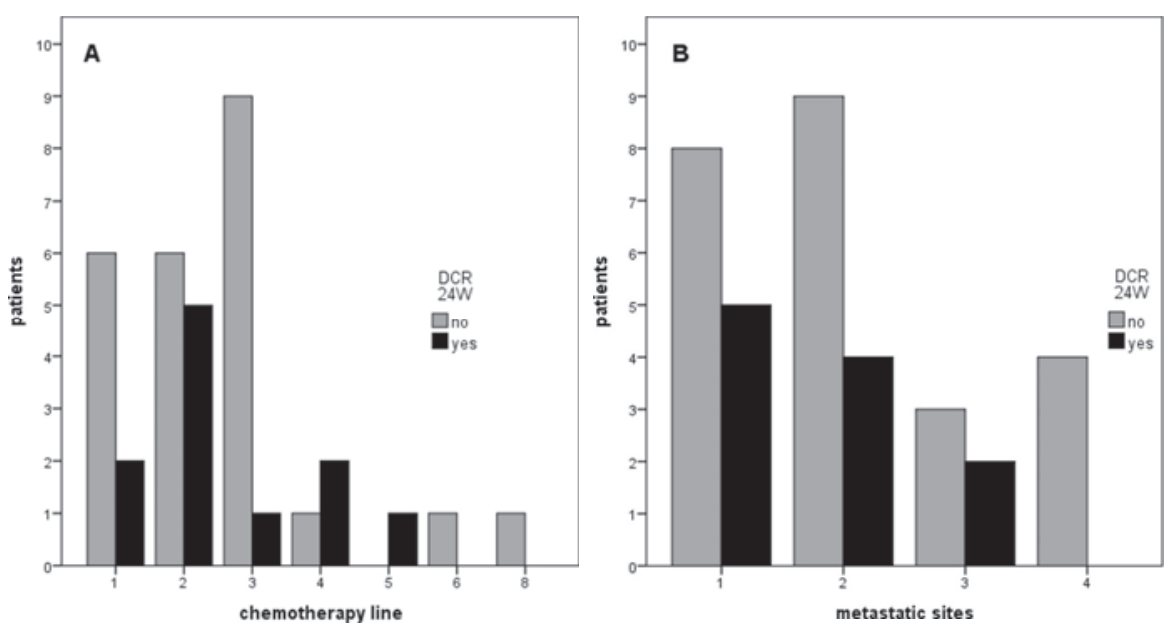

Fig. 1. Treatment response (disease control rate $\geq 24$ weeks, DCR $24 \mathrm{~W}$ ) with regard to subgroups: A number of chemotherapy lines; $\mathbf{B}$ number of different metastatic sites; $\mathbf{C}$ hormone receptor (HR) status; D HER2 status.
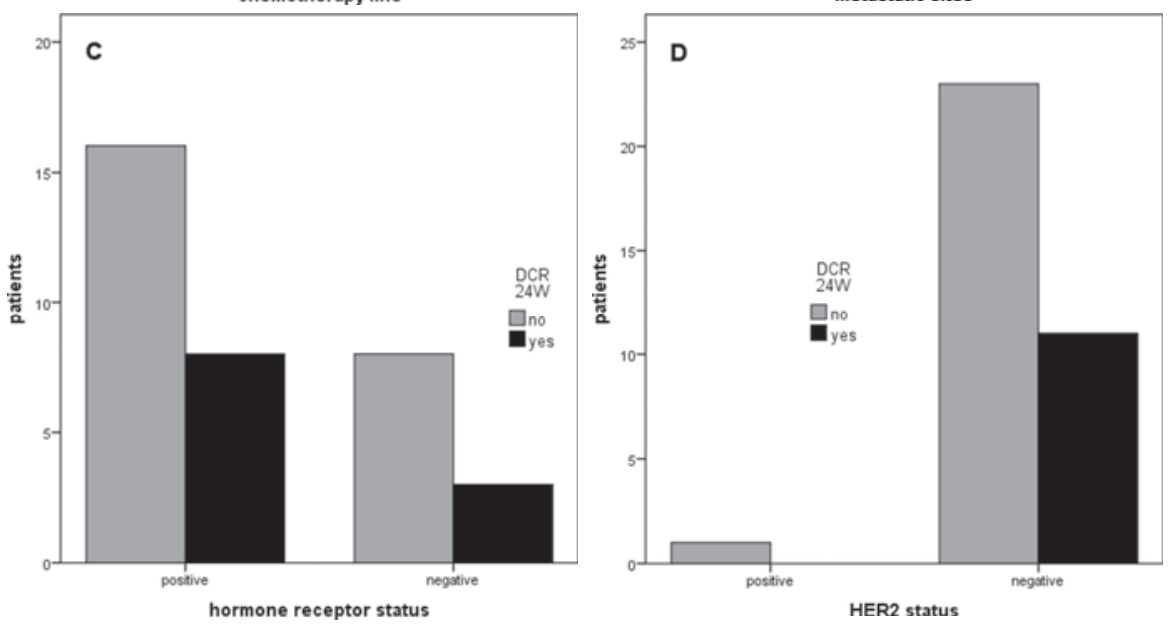

Table 5. Treatment response (disease control rate $\geq 24$ weeks) with regard to subgroups

\begin{tabular}{lcc}
\hline Subgroup & Rate, $\mathrm{n}(\%)$ & $\mathrm{p}$ value \\
\hline DCR depending on chemotherapy line & & 0.493 \\
$\quad \leq 2$ & $7 / 19(36.8)$ & \\
$\quad>2$ & $4 / 16(25)$ & \\
DCR depending on number of metastatic sites & & 0.685 \\
$\quad \leq 2$ & $9 / 26(34.6)$ & \\
$\quad>2$ & $2 / 9(22.2)$ & \\
DCR depending on hormone receptor status & & 1.00 \\
$\quad$ Positive & $8 / 24(33.3)$ & \\
$\quad$ Negative & $3 / 11(27.3)$ & \\
DCR depending on HER2 status & & - \\
$\quad$ Positive & $0 / 1(0)$ & \\
$\quad$ Negative & $11 / 34(32.4)$ & \\
\hline
\end{tabular}

ily pretreated and $7 / 19$ (37\%) non-heavily pretreated patients achieved DCR ( $\mathrm{p}=0.493) .2 / 9(22 \%)$ patients with multiple metastases and $9 / 26(35 \%)$ with $\leq 2$ different metastatic sites achieved DCR ( $\mathrm{p}=0.685$ ). 8/24 (33\%) HR-positive and 3/11 (27\%) HR-negative patients achieved DCR $(\mathrm{p}=1.00)$. We did not conduct a comparison regarding HER2 status and therapy response due to the lack of HER2-positive patients (HER2-positive: $\mathrm{n}=1$; HER2-negative: $\mathrm{n}=34$ ).

\section{Discussion}

In this explorative analysis of 35 heavily pretreated MBC patients, LDMC was efficient and well tolerated. The treated cohort varied in age, number of pretreatments, and number of metastatic sites before the start of LDMC. The DCR of $31 \%$ was in line with the results of previous phase II studies in which the DCR/clinical benefit rate (CBR) was achieved in $24-51 \%$ of patients $[10,11,14]$. $\mathrm{CR}, \mathrm{PR}$, and SD were observed in 1,6 , and 4 patients, respectively. The median PFS was 12 weeks (range 6-86 weeks), and the median DoR was 22 weeks (range 8-74 weeks). Orlando et al. [15] showed in a long-term follow-up study that metronomic CTX/MTX was feasible and provided a prolonged clinical benefit (CB) in $16 \%$ of patients without cumulative toxicity despite prolonged use. In a prospective, non-randomized, phase II clinical trial, Perroud et al. [12] showed in patients with advanced breast cancer a CB of $55 \%$ $(11 / 20)$ at 24 weeks after the beginning of treatment with metronomic CTX and celecoxib, a selective cyclooxygenase- 2 inhibitor. Moreover, serum concentration of vascular endothelial growth factor (VEGF) decreased and soluble vascular endothelial growth factor receptor 2 (sVEGFR-2) increased during treatment. Circulating endothelial cells (CECs) increased in patients with $\mathrm{CB}$ at the time of progression. Perroud et al. [12] postulated baseline VEGF and VEGF/sVEGFR-2 to be potential predictive biomarkers of re- 
sponse, and CECs of follow-up, in metronomic chemotherapy. Because of the well-known anti-angiogenic effects of LDMC, combinations with other agents targeting VEGF were evaluated [16, 17]. A phase II trial with metronomic CTX and capecitabine in combination with bevacizumab showed a CBR of $68 \% \geq 24$ weeks and a mild toxicity profile in heavily pretreated MBC patients [18]. The orally available VEGFR inhibitor, vandetanib, given metronomically with metronomic CTX/MTX, obtained a favorable therapy response (DCR of $25 \% \geq 24$ weeks) with a good toxicity profile in MBC [19].

LDMC with CTX/MTX was well tolerated. 7 (20\%) patients discontinued therapy due to adverse events. 3 (9\%) patients stopped both CTX and MTX therapy due to intolerable side effects. Thrombocytopenia, gastrointestinal complaints, and fatigue were the most common reasons for early termination of therapy. 4 (11\%) patients stopped MTX but continued CTX, mainly because of gastrointestinal symptoms and vision impairment. 1 (3\%) patient developed hemorrhagic cystitis but no therapy interruption was necessary. Leukopenia and anemia were not documented as reasons for therapy termination. In the study by Colleoni et al. [10], MBC patients with Eastern Cooperative Oncology Group (ECOG) scores $\leq 3$ treated with metronomic CTX/MTX presented with few adverse events. The most frequent toxicity was grade 1 leukopenia, which was observed in $35 \%$ of cases. Only $10 \%$ of the cycles were delayed and $7 \%$ of the courses were administered at reduced dosages, mainly due to leukopenia and an increase in transaminases [10].

In our subgroup analysis, no significant differences were found regarding chemotherapy lines, number of different metastatic sites, and HR status. Not surprisingly, we observed that therapy response was worse with an increasing number of metastatic sites and chemotherapy lines. It can be assumed that the LDMC should be investigated not only in heavily pretreated patients but also in MBC patients without symptoms and need for rapid response. Recently, LDMC was also examined as a component of the adjuvant and neoadjuvant treatment of breast cancer [9, 20,21].

LDMC should be discussed as a feasible low-dose variation of dose-dense therapy. Compared to MTD chemotherapy, LDMC is associated with significantly lower cumulative doses and less toxicity $[1,2,6,21]$. Despite this finding, the antitumor effects of LDMC might be comparable or even superior to conventional MTD regi- mens [22-24]. The main efficacy of LDMC is induced by anti-angiogenesis and immune system modulation [25-29]. Furthermore, LDMC exerts inhibiting effects on the tumor and, in contrast to MTD regimens, also on tumor-initiating cells [30, 31].

The main experience with LDMC in MBC arises from phase II studies. The most frequently administered therapy consists of CTX and MTX [10, 11, 13, 14, 32, 33]; however, prospective randomized studies are lacking [7]. Other orally available drugs with proven efficacy in MBC, like vinorelbine or capecitabine, also showed high potential in metronomic schedules $[34,35]$. Moreover, there is still a lack of randomized studies of LDMC in comparison to approved chemotherapies to determine the exact role of LDMC in the palliative treatment of MBC patients. To evaluate the efficacy and advantages of LDMC in comparison with conventional chemotherapy in the palliative treatment of $\mathrm{MBC}$, we have initiated a casecontrol study. The retrospective design of the current analysis limited the examination of further important aspects like quality of life. In the next step, we will prepare a prospective case-control analysis of LDMC and conventional chemotherapy to examine subgroups and determine the patient collective which will obtain the best benefit from LDMC in the treatment of MBC.

\section{Conclusion}

In conclusion, our retrospective analysis of metronomic CTX/ MTX indicates that LDMC is a feasible and well tolerated therapy option in MBC patients without the need for rapid response. However, effectiveness and tolerability should be examined in prospective randomized trials.

\section{Acknowledgement}

Parts of the presented results derive from the doctoral thesis of Ms. Sara Plavic-Radeka.

\section{Disclosure Statement}

The authors state that there are no conflicts of interest to declare.

\section{References}

1 Hanahan D, Bergers G, Bergsland E: Less is more, regularly: metronomic dosing of cytotoxic drugs can target tumor angiogenesis in mice. J Clin Invest 2000;105 1045-1047.

2 Banys-Paluchowski M, Schutz F, Ruckhaberle E, Krawczyk N, Fehm T: Metronomic chemotherapy for metastatic breast cancer - a systematic review of the literature. Geburtshilfe Frauenheilkd 2016;76:525-534.

3 Kontani K, Hashimoto SI, Murazawa C, et al.: Indication of metronomic chemotherapy for metastatic breast cancer: clinical outcomes and responsive subtypes. Mol Clin Oncol 2016;4:947-953.

\footnotetext{
4 Hortobagyi GN: Treatment of breast cancer. N Engl J Med 1998;339:974-984.

5 Cardoso F, Costa A, Senkus E, et al.: 3rd ESO-ESMO international consensus guidelines for advanced breast cancer (ABC 3). Breast 2017;31:244-259.

6 Montagna E, Cancello G, Dellapasqua S, Munzone E, Colleoni M: Metronomic therapy and breast cancer: a systematic review. Cancer Treat Rev 2014;40:942-950.

7 Colleoni M, Orlando L, Sanna G, et al.: Metronomic low-dose oral cyclophosphamide and methotrexate plus or minus thalidomide in metastatic breast cancer: antitumor activity and biological effects. Ann Oncol 2006; 17:232-238.
}

8 Munzone E, Colleoni M: Clinical overview of metronomic chemotherapy in breast cancer. Nat Rev Clin Oncol 2015; 12:631-644.

9 Colleoni M, Gray KP, Gelber S, et al.: Low-dose oral cyclophosphamide and methotrexate maintenance for hormone receptor-negative early breast cancer: International Breast Cancer Study Group trial 22-00. J Clin Oncol 2016;34:3400-3408

10 Colleoni M, Rocca A, Sandri MT, et al.: Low-dose oral methotrexate and cyclophosphamide in metastatic breast cancer: antitumor activity and correlation with vascular endothelial growth factor levels. Ann Oncol 2002;13:73-80. 
11 Gebbia V, Boussen H, Valerio MR: Oral metronomic cyclophosphamide with and without methotrexate as palliative treatment for patients with metastatic breast carcinoma. Anticancer Res 2012;32:529-536.

12 Perroud HA, Alasino CM, Rico MJ, et al.: Metastatic breast cancer patients treated with low-dose metronomic chemotherapy with cyclophosphamide and celecoxib: clinical outcomes and biomarkers of response. Cancer Chemother Pharmacol 2016;77:365374.

13 Wong NS, Buckman RA, Clemons M, et al.: Phase I/I trial of metronomic chemotherapy with daily dalteparin and cyclophosphamide, twice-weekly methotrexate, and daily prednisone as therapy for metastatic breast cancer using vascular endothelial growth factor and soluble vascular endothelial growth factor receptor levels as markers of response. J Clin Oncol 2010;28: 723-730.

14 Miscoria M, Tonetto F, Deroma L, et al.: Exploratory predictive and prognostic factors in advanced breast cancer treated with metronomic chemotherapy. Anticancer Drugs 2012;23:326-334

15 Orlando L, Cardillo A, Rocca A, et al.: Prolonged clinical benefit with metronomic chemotherapy in patients with metastatic breast cancer. Anticancer Drugs 2006; 17:961-967.

16 Montagna E, Cancello G, Bagnardi V, et al.: Metronomic chemotherapy combined with bevacizumab and erlotinib in patients with metastatic HER2-negative breast cancer: clinical and biological activity. Clin Breast Cancer 2012;12:207-214.

17 Riesco-Martinez M, Parra K, Saluja R, Francia G, Emmenegger U: Resistance to metronomic chemotherapy and ways to overcome it. Cancer Lett 2017; 400:311-318.

18 Dellapasqua S, Bertolini F, Bagnardi V, et al.: Metronomic cyclophosphamide and capecitabine combined with bevacizumab in advanced breast cancer. J Clin Oncol 2008;26:4899-4905.
19 Mayer EL, Isakoff SJ, Klement G, et al.: Combination antiangiogenic therapy in advanced breast cancer: a phase 1 trial of vandetanib, a VEGFR inhibitor, and metronomic chemotherapy, with correlative platelet proteomics. Breast Cancer Res Treat 2012;136:169178.

20 Munzone E, Colleoni M: Metronomics in the neoadjuvant and adjuvant treatment of breast cancer. Cancer Lett 2017;400:259-266.

21 Romiti A, Falcone R, Roberto M, Marchetti P: Current achievements and future perspectives of metronomic chemotherapy. Invest New drugs 2017;35:359-374.

22 Bertolini F, Paul S, Mancuso P, Monestiroli S, Gobbi A, Shaked Y, Kerbel RS: Maximum tolerable dose and low-dose metronomic chemotherapy have opposite effects on the mobilization and viability of circulating endothelial progenitor cells. Cancer Res 2003;63:43424346.

23 Kerbel RS, Kamen BA: The anti-angiogenic basis of metronomic chemotherapy. Nat Rev Cancer 2004;4: 423-436.

24 Mavroeidis L, Sheldon H, Briasoulis E, Marselos M, Pappas P, Harris AL: Metronomic vinorelbine: antiangiogenic activity in vitro in normoxic and severe hypoxic conditions, and severe hypoxia-induced resistance to its anti-proliferative effect with reversal by Akt inhibition. Int J Oncol 2015;47:455-464.

25 Browder T, Butterfield CE, Kraling BM, Shi B, Marshall B, O’Reilly MS, Folkman J: Antiangiogenic scheduling of chemotherapy improves efficacy against experimental drug-resistant cancer. Cancer Res 2000;60: 1878-1886.

26 Ghiringhelli F, Menard C, Puig PE, et al.: Metronomic cyclophosphamide regimen selectively depletes $\mathrm{CD} 4+\mathrm{CD} 25+$ regulatory $\mathrm{T}$ cells and restores $\mathrm{T}$ and $\mathrm{NK}$ effector functions in end stage cancer patients. Cancer Immunol Immunother 2007;56:641-648.

27 Pasquier E, Kavallaris M, Andre N: Metronomic chemotherapy: new rationale for new directions. Nat Rev Clin Oncol 2010;7:455-465.
28 Andre N, Tsai K, Carre M, Pasquier E: Metronomic chemotherapy: direct targeting of cancer cells after all? Trends Cancer 2017;3:319-325

29 Kareva I, Waxman DJ, Lakka Klement G: Metronomic chemotherapy: an attractive alternative to maximum tolerated dose therapy that can activate anti-tumor immunity and minimize therapeutic resistance. Cancer Lett 2015;358:100-106.

30 Chan TS, Hsu CC, Pai VC, et al.: Metronomic chemotherapy prevents therapy-induced stromal activation and induction of tumor-initiating cells. J Exp Med 2016;213:2967-2988.

31 Folkins C, Shaked Y, Man S, Tang T, Lee CR, Zhu Z, Hoffman RM, Kerbel RS: Glioma tumor stem-like cells promote tumor angiogenesis and vasculogenesis via vascular endothelial growth factor and stromal-derived factor 1. Cancer Res 2009;69:7243-7251.

32 Khan OA, Blann AD, Payne MJ, et al.: Continuous low-dose cyclophosphamide and methotrexate combined with celecoxib for patients with advanced cancer. Br J Cancer 2011;104:1822-1827.

33 Aurilio G, Munzone E, Botteri E, et al.: Oral metronomic cyclophosphamide and methotrexate plus fulvestrant in advanced breast cancer patients: a monoinstitutional case-cohort report. Breast J 2012;18:470474.

34 Cazzaniga ME, Camerini A, Addeo R, et al.: Metronomic oral vinorelbine in advanced breast cancer and non-small-cell lung cancer: current status and future development. Future Oncol 2016;12:373-387.

35 Cazzaniga ME, Cortesi L, Ferzi A, et al.; VICTOR Study Group: Metronomic chemotherapy with oral vinorelbine (mVNR) and capecitabine (mCAPE) in advanced HER2-negative breast cancer patients: is it a way to optimize disease control? Final results of the VICTOR-2 study. Breast Cancer Res Treat 2016;160: 501-509. 